\title{
Simulación del sistema eléctrico de potencia de nanosatélites por medio de PSpice
}

Simulation of the electrical power system of nanosatellites using PSpice

\section{Catalina Varela González ${ }^{1}$}

Varela-González, C. Simulación del sistema eléctrico de potencia de nanosatélites por medio de PSpice. Tecnología en marcha. Edición especial Movilidad Estudiantil 7. Abril, 2020. Pág. 20-35.

doi) https://doi.org/10.18845/tm.v33i6.5165 


\section{Palabras clave}

Sistema Eléctrico de Potencia; Nanosatélite; Satélite Lean; PSpice; Simulación.

\section{Resumen}

El concepto de "lean satellite" ha tomado fuerza acompañado con el incremento de pequeñas empresas y universidades que desarrollan este tipo de satélites. La construcción de satélites basada en este concepto ha permitido a estas entidades alcanzar tecnologías espaciales a pequeña escala. La creciente popularidad tiene que ver con su reducido tamaño, ligero peso y significativa menor inversión.

El sistema eléctrico de potencia provee energía a todas las cargas y subsistemas presentes en los nanosatélites. En este sistema se produce, almacena, controla y distribuye dicha energía. La comprensión de su funcionamiento resulta fundamental para asegurar el éxito de las misiones de los nanosatélites. Los componentes encargados de controlar y regular esta energía son los convertidores CC-CC que elevan o reducen la tensión de acuerdo a la demanda o al estado de tensión de la batería, por esta razón los convertidores también aseguran el correcto funcionamiento del sistema.

Este artículo se basa en el informe de proyecto de graduación titulado: Simulación del sistema eléctrico de potencia de la constelación de nanosatélites del proyecto BIRDS. Se muestra la estructura general del sistema eléctrico de potencia empleado, así como una comparación de la simulación por medio de PSpice y el funcionamiento de los convertidores CC-CC utilizados para el proyecto BIRDS 2, desarrollado en LaSEINE, laboratorio del Instituto Tecnológico de Kyushu.

\section{Keywords}

Electrical Power System; Nanosatellite; Lean Satellite; PSpice; Simulation.

\section{Abstract}

The concept of "lean satellite" has taken force accompanied by the increase of small companies and universities that develop this kind of satellites. The construction of satellites based on this concept has allowed these entities to reach space technologies on a small scale. The growing popularity has to do with their small size, light weight and significant lower investment.

The electrical power system provides power to all the loads and subsystems present in the nanosatellites. This energy is produced, stored, controlled and distributed in this system. The understanding of its operation is fundamental to ensure the success of the missions of the nanosatellite. The components responsible for controlling and regulating this energy are the DC-DC converters that boost or buck voltage according to load demand or charge of the battery, for this reason converters also ensure the correct operation of the system.

This article is based on the graduation project report titled: Simulation of the power electrical system of the nanosatellite constellation of the BIRDS project. The general structure of the power system used is shown in this article, as well as a comparison between the simulation using PSpice and the operation of the DC-DC converters used for the BIRDS 2 project, developed in LaSEINE, laboratory of Kyushu Institute of Technology. 


\section{Introducción}

El concepto de "lean satellite" se introduce para dar a entender una nueva filosofía para el desarrollo de satélites a pequeña escala, se dice que "es un satélite que utiliza un desarrollo y enfoques de gestión no tradicionales y arriesgados, con el objetivo de proporcionar al cliente valor de algún tipo, a bajo costo y sin tomar mucho tiempo para realizar la misión del satélite." [1]

Los proyectos BIRDS buscan que los estudiantes involucrados comprendan las tecnologías del espacio por medio de la construcción de los "lean satellites". En estos proyectos el tiempo de diseño y construcción es limitado, por lo que se busca ayuda de entidades externas en algunos de los procesos de diseño y construcción que disminuyen el tiempo y costos de producción. El problema es que no existe un sistema de verificación y validación eficiente para estos componentes, como en este caso del sistema eléctrico de potencia.

El sistema eléctrico de potencia para los satélites es el conjunto de elementos que cumple la función de generar, guardar, controlar y distribuir energía con la tensión de banda especificada para el bus y las cargas. Por lo general, los componentes básicos del sistema de potencia son: el panel solar, el controlador del panel solar, la batería, los reguladores de carga y descarga, el regulador de voltaje del bus, los interruptores de carga, los fusibles y el arnés de distribución. [2]

Para la regulación se utilizan convertidores de potencia CC-CC que se encargan de transformar el nivel de tensión entre dos componentes, ya sea mediante la elevación o reducción de la misma. [4] La unidad de distribución de potencia también contiene elementos importantes que se utilizan para conectar la plataforma eléctrica de cargas hacia el bus principal y en la protección al exceso de corriente. [5]

"La simulación es el proceso de diseñar un modelo de un sistema real y realizar experimentos con él para entender su comportamiento y evaluar sus especificaciones." [3] Normalmente se realizan simulaciones para el diseño de los lazos de control sobre la energía proveniente del panel solar, utilizando herramientas como MATLAB. Pero estas simulaciones no buscan el modelar con precisión los dispositivos utilizados en el diseño de circuitos integrados sino que se enfocan en teoría de circuitos, diseño de filtros, sistemas de control.

La elaboración de la simulación del sistema eléctrico de potencia utilizando PSpice, para los nanosatélites del proyecto BIRDS 2, pretende contribuir con el acceso a los modelos de los dispositivos reales utilizados en este proyecto para satisfacer la necesidad de diseñar un sistema de verificación del circuito que sea efectivo, fácil de manejar, y que además permita visualizar de forma estructurada el diseño.

Las simulaciones son parte importante en los procesos de diseño de las grandes empresas debido a la disminución de costos y tiempos de fabricación, por la misma razón se debe buscar su implementación en las investigaciones con fines educativos. La publicación de este artículo pretende incentivar el desarrollo de nuevos sistemas de verificación y validación utilizando herramientas computacionales.

El objetivo principal del presente artículo es determinar la validez de la simulación de los componentes del sistema eléctrico de potencia como instrumento de prueba y análisis del circuito físico. El artículo consta de tres partes principales, en la primera parte se explica el método utilizado para obtener los resultados que se muestran y analizan en la segunda parte, y finalmente en la tercera parte se presentan las conclusiones. 


\section{Metodología}

En primer lugar se establece el flujo de trabajo y estructura básica del sistema eléctrico de potencia de los satélites del proyecto BIRDS 2. La figura 1 muestra como el proceso comienza al tomar la energía solar por medio de los paneles, esta se regula antes de permitir la alimentación del sistema de carga. Seguidamente, se añade la batería, que se encargará de almacenar y abastecer la energía. A partir de este punto, se realiza la distribución de energía, no sin antes añadir protecciones en caso de que exista un exceso de corriente en el sistema. Esta distribución cuenta con energía regulada y sin regular que abastecerá cada sistema dependiendo de las demandas y limitaciones.

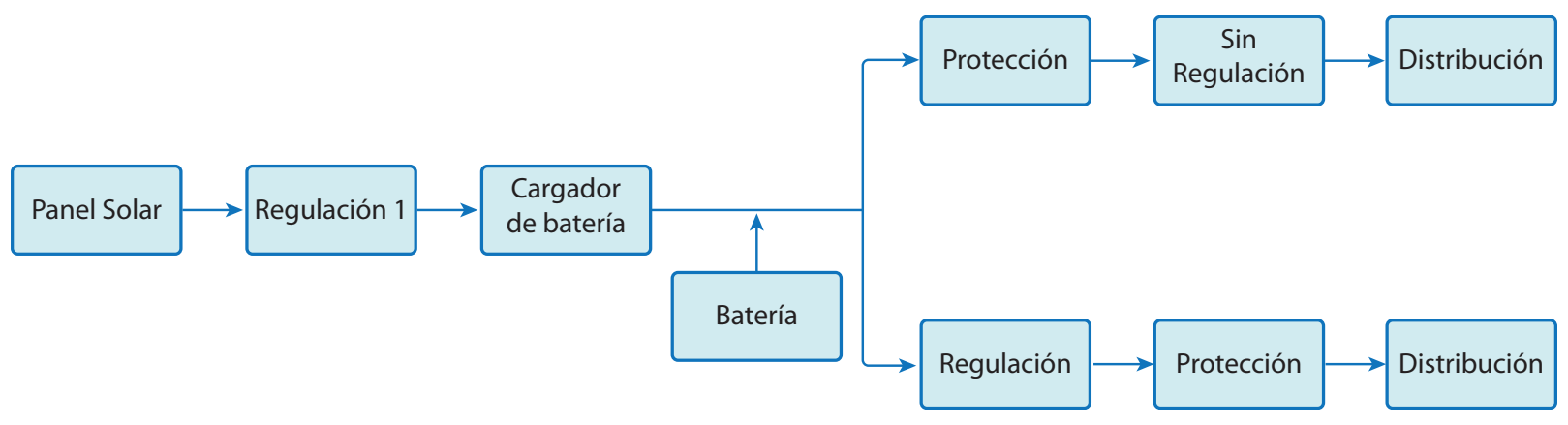

Figura 1. Diagrama de bloques de alto nivel del sistema eléctrico de potencia. Elaboración propia utilizando Lucidchart.

Para seleccionar los parámetros de interés, se estudian todos los elementos del sistema frente a situaciones críticas de nivel de carga de la batería y la demanda de potencia exigida por la carga del sistema, esto se define al considerar que dichas condiciones no deben alterar el funcionamiento del sistema de potencia porque esto significaría una falla en los subsistemas abastecidos. De esta forma, se eligen las tensiones de salida de los convertidores TPS63020 a 3.3V y el TPS61235 a 5V, y se desea comprobar que a distintas condiciones de la batería (alta y baja tensión), el sistema sea capaz de mantener la tensión de salida establecida.

Otra de las características críticas es la demanda energética del sistema. De esta manera, se busca analizar la respuesta de los convertidores escogidos frente a grandes cargas, simulando una eventual sobrecarga al sistema. Es por este motivo, que se busca determinar los límites hasta los cuáles cada convertidor funciona de forma adecuada.

Para desarrollar la simulación lo más cercano al sistema real, se decide simular el panel solar de acuerdo a los modelos ya existentes, se añade el modelo más sencillo de una batería y se añaden los distintos componentes al sistema de acuerdo a su configuración. En primer lugar, se divide la simulación en cada uno de los componentes y se comprueba que la configuración individual se comporte de la misma manera que lo hace el dispositivo en la realidad. A partir de esto, se desea unir cada uno de los componentes en bloques más grandes hasta integrar un sistema.

En el caso del panel solar, se utiliza como guía el modelo que presenta Patel. [2] De esta forma se obtienen los valores de las resistencias y se le asignan características específicas al diodo del modelo. Con esto se pretende comparar la corriente $\mathrm{I}_{\mathrm{SC}}$ y la tensión $\mathrm{V}_{\mathrm{OC}}$ del modelo contra los datos presentados en la hoja de datos de la celda solar TJ3G30A. Cada una de las baterías que poseen una tensión nominal de $1.2 \mathrm{~V}$, se simulan utilizando una fuente de tensión constante y una resistencia cuyo valor es obtenido por medio de las pruebas de seguridad realizadas previamente en el laboratorio. 
Para cumplir con las normativas y los procesos de seguridad exigidos por la Agencia Japonesa de Exploración Aeroespacial (JAXA), se añaden distintos dispositivos empleados para asegurar el correcto encendido y apagado del sistema en los momentos que se requieren. En todos los casos se utiliza un circuito básico de prueba para conmutadores tipo MOSFET, que corresponde a la alimentación del pin Gate con una señal periódica de encendido y apagado, la conexión de una fuente de tensión CC y una carga al pin de Source y finalmente el pin Drain conectado a tierra.

En la figura 2 se muestra el sistema final, una vez que se han realizado las conexiones entre los componentes configurados por medio de la herramienta presente en el software la cual permite ramificar el sistema e integrarlo en una vista de esquemático final que se comportará como el programa principal. De este modo, los complejos circuitos serán visualizados como cajas con entradas y salidas que brindan una apariencia más limpia y sencilla. Y la simulación de la energía consumida por el sistema, se realiza por medio de una fuente de corriente que definirá los cambios en la demanda de energía del sistema y que se puede comparar con la carga electrónica propuesta para medir el sistema real.

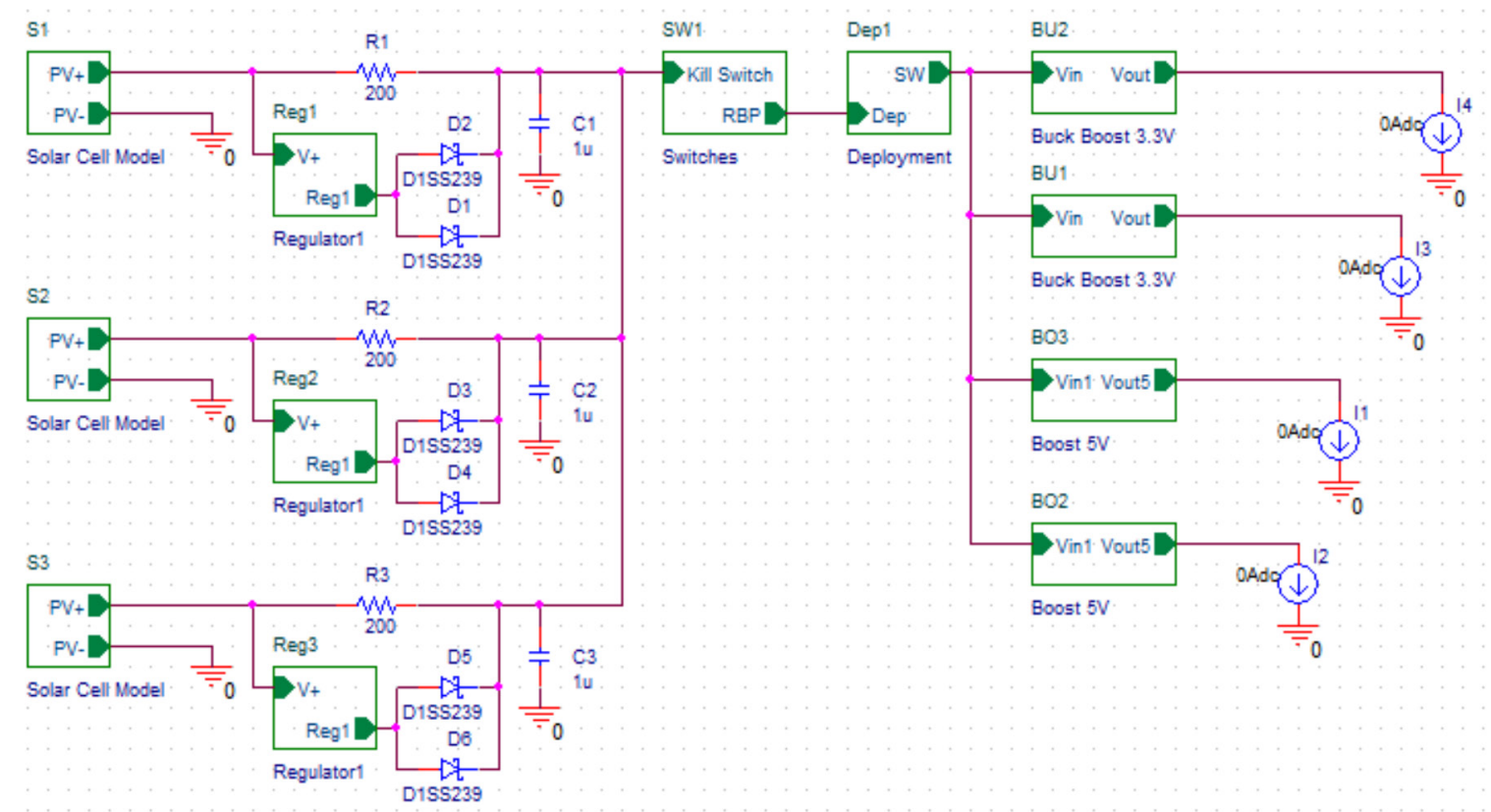

Figura 2. Simulación del sistema eléctrico de potencia. Elaboración propia utilizando PSpice.

\section{Métodos para la Medición del Modelo de Ingeniería}

Para realizar las mediciones se establecen dos métodos en los cuáles se medirán las salidas reguladas de los convertidores TPS61235 y TPS63020 en condiciones distintas de operación, como la demanda de corriente por parte de los sistemas a los que se distribuye la energía y la carga de la batería. 


\section{Método 1}

Como se menciona anteriormente, este método no invasivo busca obtener el valor de la tensión de salida de los convertidores TPS61235 y TPS63020 por medio el ADC AD7298 que recolecta los datos dentro del sistema eléctrico de potencia y transmitirlos por medio de comunicación serial a través del programador diseñado para BIRDS 2 que consiste principalmente en el componente ADM3202. Las baterías de Hidruro de Níquel Metal, se encargan de alimentar las placas a $4.012 \mathrm{~V}$ y no se le aplica ninguna carga externa. Utilizando los componentes y el equipo que se enlista en el cuadro 1, se verifica la tensión para el suministro de 3.3V.

Componentes y Equipo

Cuadro 1. Componentes y equipo utilizados para el método 1 de medición.

\begin{tabular}{|l|c|}
\hline Nombre & Cantidad \\
\hline Sistema Eléctrico de Potencia BIRDS 2 & 1 \\
\hline Programador para OBC de BIRDS 2 & 1 \\
\hline Cable de conexión serial & 1 \\
\hline Programa computacional Putty & 1 \\
\hline Guantes de látex & 1 \\
\hline
\end{tabular}

Procedimiento

El primer paso será acondicionar el lugar de trabajo, seleccionando un área libre en el que se pueda colocar de forma segura el modelo de ingeniería, además se debe utilizar guantes para manipular el sistema de forma segura. En el circuito de prueba, los interruptores de seguridad se encuentran de tal forma que únicamente es necesario remover el "Kill Switch" para permitir que las baterías comiencen a energizar el sistema eléctrico de potencia, por lo que el primer paso será identificar y remover este pin.

En seguida, se puede iniciar la comunicación serial a través del software de monitoreo serial de preferencia, en este caso se utiliza Putty en éste se despliegan inmediatamente las configuraciones iniciales, y al presionar la tecla de entrada se visualiza el menú con las actividades que pueden ser realizadas por medio del programador. Las opciones son: a: Get Housekeeping data, b: EPS Power output control, c: Housekeeping data collection, d: Check Flash Memories, h: See satellite Log, f: Reset All Time to 0, i: SEL current Measurement, m: Battery measurement, r: H8 Com Reset, t: Fuctional test, k: Command Execution.

Por medio de la letra "b", se debe asegurar que los pines de suministro que se desean medir SUP 3.3V y SUP 5.0V se encuentren encendidos, esta opción guiará al usuario para que pueda realizar dicha acción. Seguidamente para visualizar los datos se debe introducir la letra "a" en el menú principal y esto nos brindará la corriente y potencia medidas, con lo que por medio de cálculos, se pueden obtener las verdaderas tensiones en estas salidas.

\section{Método 2}

Este método consiste en probar directamente los convertidores TPS61235 y TPS63020 que proveen SUP 3.3V y SUP 5V. En este caso, se utilizará una fuente de tensión de corriente continua en lugar de las baterías, con tensiones de $3 \mathrm{~V}$ y $4.2 \mathrm{~V}$, esto con el fin de simular dos estados críticos en la batería. A la salida de estos convertidores se colocará una carga eléctrica con la cual se regulará la corriente demandada por los elementos que se conectan a los convertidores mencionados y se medirá desde 0 hasta el punto en el que el circuito de protección de potencia entra en funcionamiento, realizando las mediciones para cada 0.050A. En el cuadro 2 se enlistan los elementos necesarios para llevar a cabo este experimento. 
Componentes y Equipo

Cuadro 2. Componentes y equipo utilizados para el método 2 de medición.

\begin{tabular}{|l|c|}
\hline \multicolumn{1}{|c|}{ Nombre } & Cantidad \\
\hline Sistema Eléctrico de Potencia BIRDS 2 & 1 \\
\hline Fuente CC GWINSTECK PSW 80-13.5 & 1 \\
\hline Carga electrónica PLZ164WA & 1 \\
\hline Multimetro & 1 \\
\hline Guantes de látex & 1 \\
\hline
\end{tabular}

\section{Procedimiento}

Se toman las mismas medidas de seguridad establecidas en el método 1. Luego se coloca la tensión de 3V o 4.2V en la terminal dónde se debe colocar la batería y se conecta la carga electrónica. Se ubican las salidas de los convertidores TPS61235 y TPS63020 y se miden por medio de un multímetro.

\section{Resultados y Análisis}

En general, se utilizarán las ecuaciones obtenidas de [6], las mismas se muestran a continuación y se utilizan para analizar los valores que corresponden respectivamente al porcentaje de error, el promedio de las mediciones, y la desviación estándar. Con ellas se busca establecer estadísticamente la validez de las mediciones realzadas y la proximidad de los datos de la simulación contra los valores medidos en los dispositivos reales.

$$
\begin{gathered}
\text { \%Error }=\frac{\text { Valor }_{\text {Real }}-\text { Valor }_{\text {Aproximado }}}{\text { Valor }_{\text {Real }}} \times 100 \\
\overline{\mathrm{x}}=\frac{\sum_{\mathrm{i}=1}^{\mathrm{n}} \mathrm{x}_{\mathrm{i}}}{\mathrm{n}} \\
S=\sqrt{\frac{\sum_{\mathrm{i}=1}^{\mathrm{n}}\left(\mathrm{x}_{\mathrm{i}}-\overline{\mathrm{x}}\right)^{2}}{\mathrm{n}-1}}
\end{gathered}
$$

En la figura 3, se muestra la curva obtenida para el panel solar modelado con los parámetros establecidos. Se observa la forma de curva teóricamente esperada para un gráfico de tensión de circuito abierto en función de la corriente de cortocircuito para un panel solar, de aquí se puede calcular la potencia extraída del panel solar, utilizando un punto de corriente y un punto de tensión y calculando el área bajo la curva resultante. 


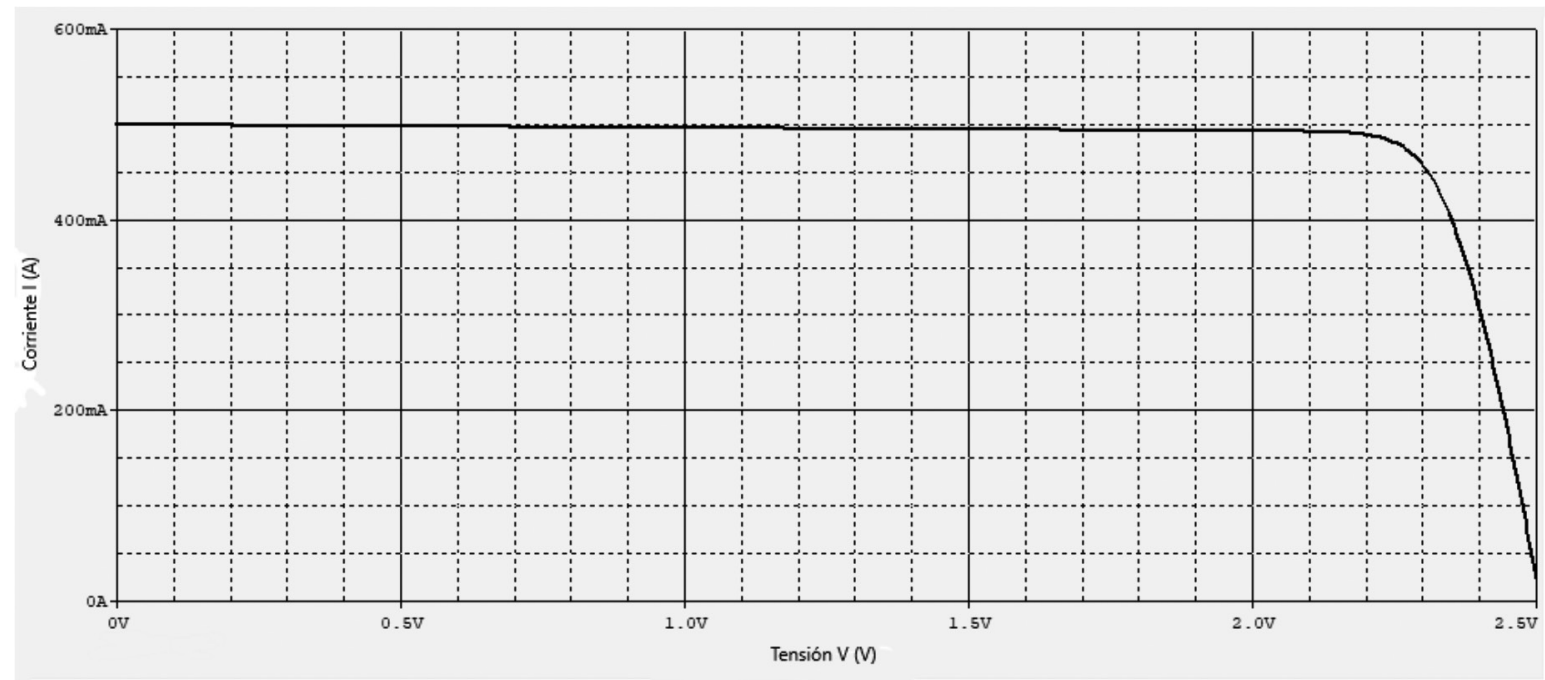

Figura 3. Simulación del gráfico $\mathrm{V}_{\mathrm{OC}}$ vs $\mathrm{I}_{\mathrm{SC}}$ de la celda solar. Elaboración propia utilizando PSpice.

Una vez identificada la forma característica de esta curva se procede a comparar los datos obtenidos en la simulación frente a los valores dados por la hoja de datos. Esta comparación permite conocer la exactitud del circuito modelado con respecto a los valores encontrados para estos paneles solares. En el cuadro 3 se presenta la comparación entre los datos del panel solar y la simulación del mismo.

Los datos obtenidos mediante la simulación se miden en el punto máximo de potencia que se extraen de la figura 3, y los datos para la comparación se extraen de la celda solar TJ3G30A. En el cuadro 3 se ubican los porcentajes de error asociados a estas mediciones, con los que se determina que en el caso de la tensión de circuito abierto existe un porcentaje de error que debe ser considerado, dependiendo del estudio que se desee realizar, sin embargo, sigue siendo bastante cercano al valor proporcionado por la hoja de datos. En el caso de la $\mathrm{I}_{\mathrm{sc}} \mathrm{su}$ exactitud es mayor, pero se debe ser cuidadoso al utilizar este tipo de modelos en simulaciones que requieran una exactitud mayor a la obtenida.

Cuadro 3. Datos eléctricos de la celda solar y los porcentajes de error asociados.

\begin{tabular}{|c|c|c|c|}
\hline Datos Eléctricos & Hoja de Datos & PSpice & Error (\%) \\
\hline Tensión de Circuito Abierto $\mathrm{V}_{\mathrm{OC}}$ & $2512 \mathrm{mV}$ & $2243 \mathrm{mV}$ & 11 \\
\hline Corriente de Cortocircuito I $_{\mathrm{SC}}$ & $501.3 \mathrm{~mA}$ & $482.9 \mathrm{~mA}$ & 6 \\
\hline
\end{tabular}

En el cuadro 4, se muestran los valores teóricos de la tensión que se configuró de acuerdo a la elección de los valores de los componentes y el valor de tensión estable obtenido por medio de la simulación por PSpice. En esta etapa se busca comprobar únicamente su funcionamiento de acuerdo a los componentes de entrada y salida establecidos, sin cambiar las condiciones de carga y batería del circuito.

Para cada uno de los comparadores se obtienen los porcentajes de error que determinan con qué exactitud la tensión de salida se comporta de acuerdo a los capacitores e inductores seleccionados, de los resultados se puede observar que el modelo en PSpice de los convertidores TPS61071, TPS61235 y TPS63020 es bastante exacto para los requerimientos comunes, excepto para el TPS62130 que pierde un poco de exactitud con un 12\% de error. 
Cuadro 4. Comparación entre los valores de tensión de los valores teóricos esperados y los obtenidos mediante PSpice

\begin{tabular}{|c|c|c|c|}
\hline Convertidor & Tensión $\mathrm{V}_{\text {。 }}$ Teórica $(\mathrm{V})$ & Tensión $\mathrm{V}_{\text {。 }}$ en PSpice $(\mathrm{V})$ & Error $(\%)$ \\
\hline TPS62130 & 4.2 & 3.7 & 12 \\
\hline TPS61071 & 5.1 & 5.199 & 2 \\
\hline TPS61235 & 5.0 & 5.068 & 1 \\
\hline TPS63020 & 3.3 & 3.277 & 0.7 \\
\hline
\end{tabular}

Con respecto a los componentes de seguridad, se muestra básicamente el cumplimiento de su funcionamiento como interruptores. Y se crea el cuadro 5, donde se muestran los valores de tensión $V_{D S}$ que cada uno de ellos permite obtener en cada tensión de $V_{G S}$ aplicada. Por lo tanto, se demuestra que efectivamente el modelo de cada uno de los MOSFET para PSpice cumple correctamente con su función de interruptor, que permite el paso y la interrupción de la tensión según el control en la tensión $V_{G S}$ que se le asigne a cada uno. Debido a que no se lleva a cabo la medición de los MOSFET físicos, no se realiza el análisis de las pérdidas de tensión que vienen asociadas a estos dispositivos.

Cuadro 5. Tensiones $\mathrm{V}_{\mathrm{DS}}$ para los MOSFET

\begin{tabular}{|c|c|c|}
\hline Interruptor & Tensión $\mathrm{V}_{\mathrm{DS}}$ con $\mathrm{V}_{\mathrm{GS}}=5 \mathrm{~V}(\mathrm{~V})$ & Tensión $\mathrm{V}_{\mathrm{DS}}$ con $\mathrm{V}_{\mathrm{GS}}=0 \mathrm{~V}(\mathrm{~V})$ \\
\hline TPS22965N & 5 & 0 \\
\hline Si706DN & 5 & 0 \\
\hline SiA447DJ & 5 & 0.8 \\
\hline SSM3K15AMFV & 5 & 0 \\
\hline SSM6K411TU & 5 & 0 \\
\hline
\end{tabular}

\section{Resultados del sistema Real}

Seguidamente, se muestran los gráficos obtenidos por medio de las mediciones realizadas al sistema real, las cuáles se repitieron seis veces para cada uno de los estados medidos con lo que se calculó un valor promedio y se comparó con los datos obtenidos en la simulación. Además, se realizan cálculos estadísticos que permiten observar la exactitud de las mediciones y las diferencias encontradas entre los dos sistemas. 


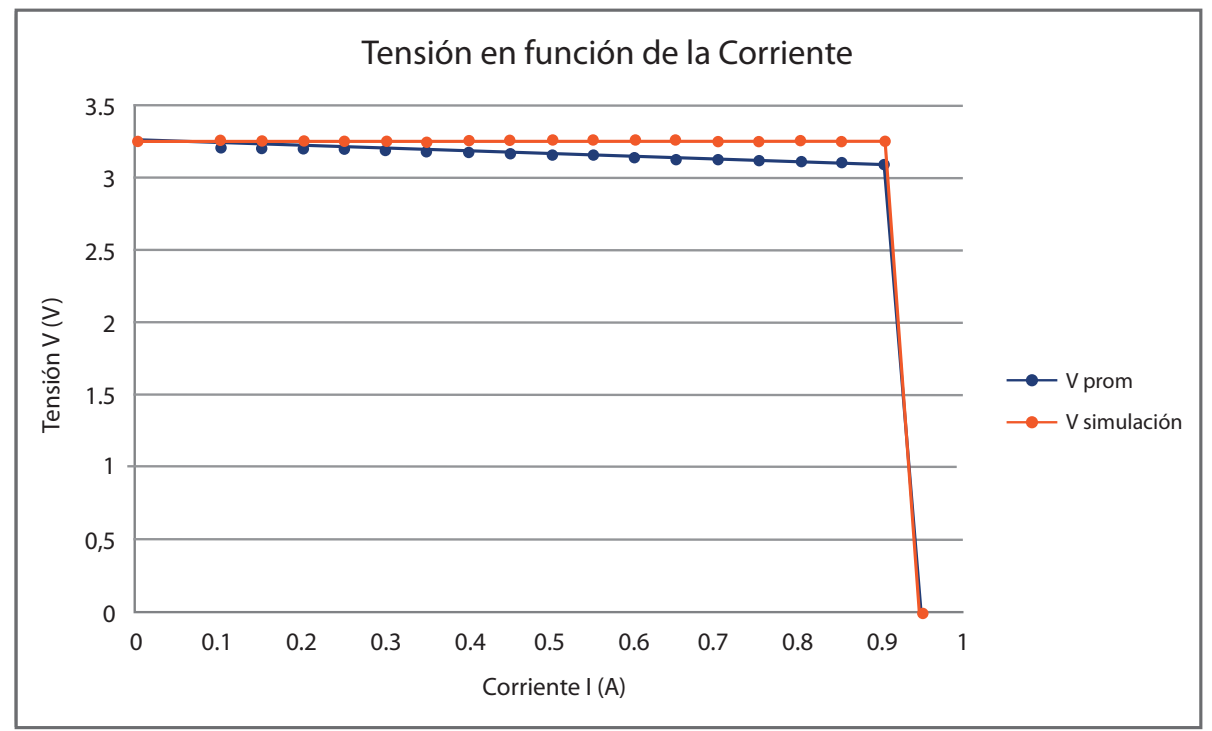

Figura 4. Convertidor TPS63020 con alimentación 4.2V. Elaboración propia utilizando Microsoft Excel.

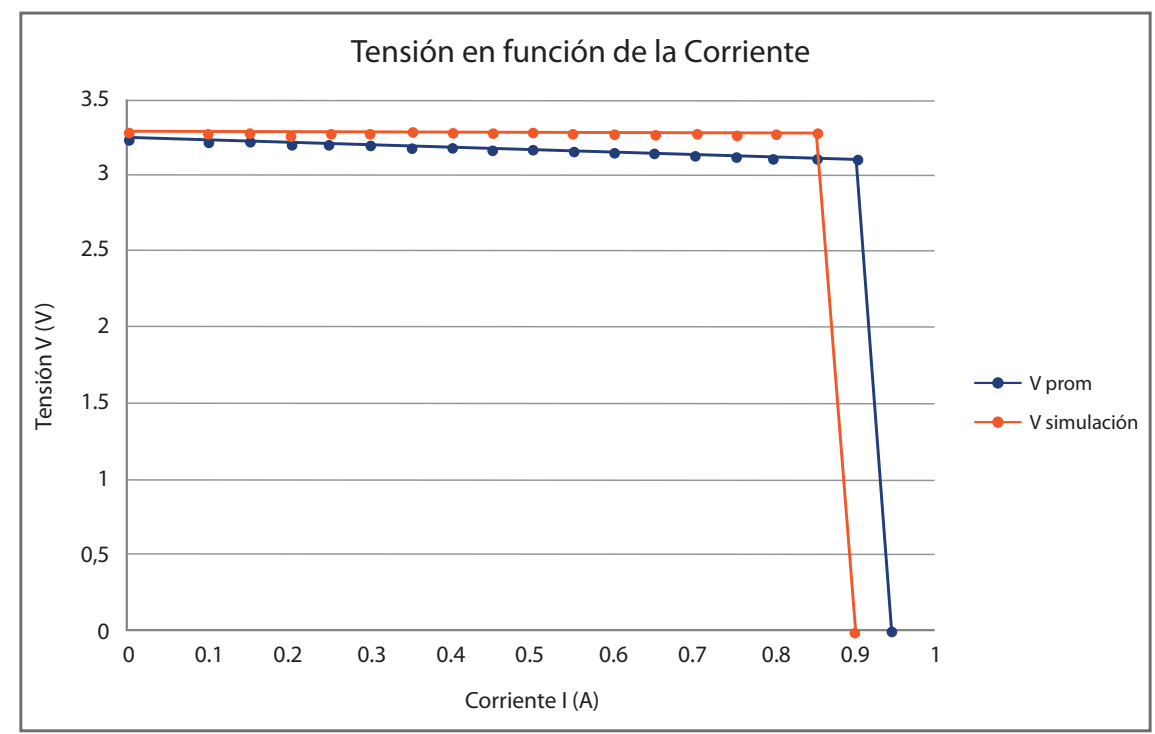

Figura 5. Convertidor TPS63020 con alimentación 3V. Elaboración propia utilizando Microsoft Excel.

Desde un punto de vista cualitativo, en el convertidor TPS63020 se puede observar que en las figuras 4 y 5 , las mediciones realizadas en el sistema real poseen un comportamiento similar a la simulación, las mediciones varían su valor con una pendiente un poco más pronunciada. Este comportamiento se mantiene para ambas tensiones de alimentación probadas y para las diferentes demandas de carga.

La mayor diferencia entre las dos tensiones de alimentación probadas es visible en la figura 5 donde la tensión cae a cero con un estado menor de carga, que limita, pero no en gran medida, el uso de la corriente en la simulación y que también podría sugerir diseñar las cargas para exigir menor corriente, y de esta forma asegurar que el componente no se encuentra cercano a sus límites y que no inducirá fallas. 


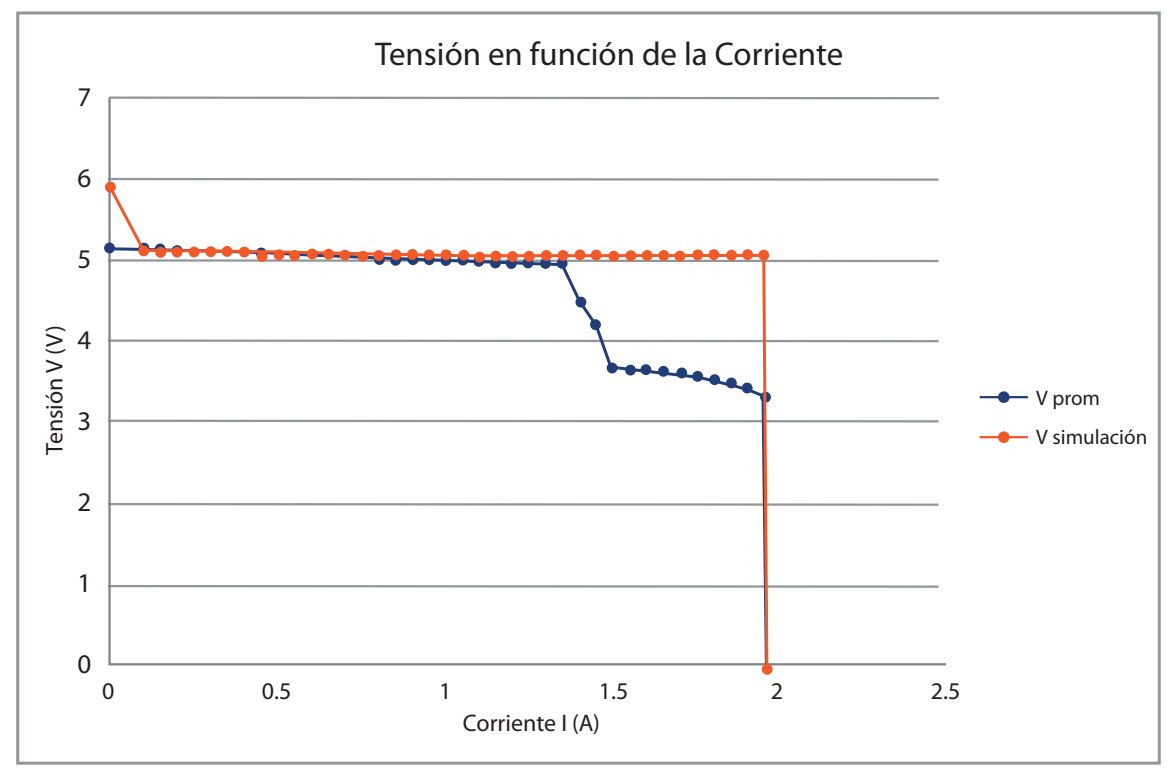

Figura 6. Convertidor TPS61235 con alimentación 4.2V. Elaboración propia utilizando Microsoft Excel.

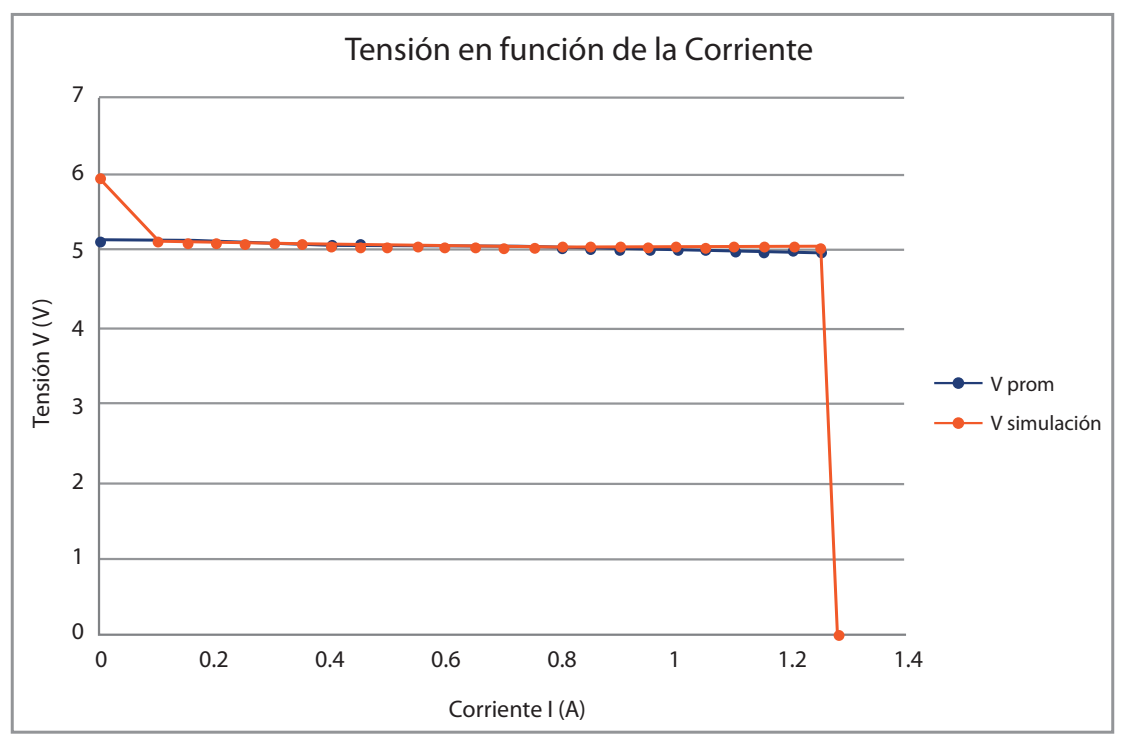

Figura 7. Convertidor TPS61235 con alimentación 3V. Elaboración propia utilizando Microsoft Excel.

En el caso del convertidor TPS61235, se observa en las figuras 6 y 7, las mediciones realizadas en el sistema real, en comparación con los datos obtenidos por medio de la simulación en PSpice. En este caso, se observa como existen espacios que poseen prácticamente la misma pendiente y donde el error es casi imperceptible de forma visual en las gráficas, sin embargo, para ambos casos se observa como el componente no reacciona de forma muy confiable al no aplicar una carga.

En el caso de la figura 7, la mayor desviación se ve reflejada al inicio, pero en el caso de la Fig. 6, se puede observar un comportamiento irregular a partir de cierta demanda de corriente por parte de la carga. La simulación continúa con la tendencia habitual pero en el componente 
físico la tensión de salida desciende mucho antes de lo supuesto. Es de esperar que para cierto rango de la gráfica, la simulación no posea la exactitud necesaria para ser utilizada.

En los cuadros 6, 7, 8 y 9, se muestran los cálculos que se representaron en las gráficas anteriores. En cada una se determina la calidad de las mediciones por medio de la desviación estándar, con la cual se observa la exactitud de las muestras que fueron tomadas, así como el porcentaje de error asociado a la comparación entre los datos obtenidos en la simulación y las mediciones del sistema real. De esta forma se comprobará de forma cuantitativa lo observado en las figuras 4, 5, 6 y 7 .

En el cuadro 6, los valores obtenidos en la simulación son bastante exactos para los estados de carga aplicados antes de que se active la protección contra el exceso de corriente y pueden ser aceptados para análisis que requieran menos de un $5 \%$ de exactitud. En el caso del cuadro 7, se observa el mismo comportamiento, pero bajo la tensión de alimentación asignada este elemento no es capaz de llegar a la tensión en la cual se desactiva el componente real, sin embargo, alcanza una condición de carga muy cercana a la real, con un porcentaje de error un poco mayor al $5 \%$.

En el cuadro 8 se observa que para el convertidor a 5.000V en la simulación es necesario aplicar una carga, y esta carga no debe superar $1.400 \mathrm{~A}$, cuando se alimenta el sistema a 4.200V, ya que a partir de este valor, el error comienza a incrementar partiendo del $13 \%$ con lo que ya no es correcto decir que la simulación se comporta de la misma forma que el componente físico.

Por otro lado, para el cuadro 9, excluyendo la condición sin carga, se obtienen porcentajes de error menores a $2 \%$, para los valores de carga inferiores a los necesarios para que el sistema real sea desconectado por la protección contra exceso de corriente.

Cuadro 6. Convertidor a 3.3V con alimentación de $4.2 \mathrm{~V}$

\begin{tabular}{|c|c|c|c|c|c|}
\hline Corriente I $(\mathrm{A})$ & $\mathrm{V}_{\text {prom }}(\mathrm{V})$ & $\mathrm{S}_{\mathrm{X}}(\mathrm{V})$ & Incertidumbre $( \pm \mathrm{V})$ & $\mathrm{V}_{\text {sim }}(\mathrm{V})$ & Error $(\%)$ \\
\hline 0.0000 & 3.269 & 0.0005164 & 0.0004 & 3.278 & 0.3 \\
\hline 0.1000 & 3.250 & 0.0005164 & 0.0004 & 3.278 & 0.9 \\
\hline 0.1500 & 3.241 & 0.0008165 & 0.0004 & 3.278 & 1 \\
\hline 0.2000 & 3.233 & 0.0005164 & 0.0004 & 3.278 & 1 \\
\hline 0.2500 & 3.224 & 0.0005164 & 0.0004 & 3.278 & 2 \\
\hline 0.3000 & 3.216 & 0.0007528 & 0.0004 & 3.277 & 2 \\
\hline 0.3500 & 3.206 & 0.0006325 & 0.0004 & 3.277 & 2 \\
\hline 0.4000 & 3.198 & 0.0007528 & 0.0004 & 3.277 & 2 \\
\hline 0.4500 & 3.191 & 0.0005164 & 0.0004 & 3.277 & 3 \\
\hline 0.5000 & 3.181 & 0.0007528 & 0.0004 & 3.276 & 3 \\
\hline 0.5500 & 3.171 & 0.0004082 & 0.0004 & 3.276 & 3 \\
\hline 0.6000 & 3.163 & 0.0005164 & 0.0004 & 3.276 & 4 \\
\hline 0.6500 & 3.153 & 0.0005164 & 0.0004 & 3.276 & 4 \\
\hline 0.7000 & 3.144 & 0.0008165 & 0.0004 & 3.275 & 4 \\
\hline 0.7500 & 3.136 & 0.0007528 & 0.0004 & 3.275 & 4 \\
\hline 0.8000 & 3.128 & 0.0005164 & 0.0004 & 3.275 & 5 \\
\hline 0.8500 & 3.120 & 0.0005164 & 0.0004 & 3.274 & 5 \\
\hline 0.9000 & 3.110 & 0.0004082 & 0.0004 & 3.274 & 5 \\
\hline 0.9460 & 0 & No aplica & 0.0004 & 3.274 & No aplica \\
\hline
\end{tabular}


Cuadro 7. Convertidor a 3.3V con alimentación de 3V

\begin{tabular}{|c|c|c|c|c|c|}
\hline Corriente I $(\mathrm{A})$ & $\mathrm{V}_{\text {prom }}(\mathrm{V})$ & $\mathrm{S}_{\mathrm{x}}(\mathrm{V})$ & Incertidumbre $( \pm \mathrm{V})$ & $\mathrm{V}_{\text {sim }}(\mathrm{V})$ & Error $(\%)$ \\
\hline 0.0000 & 3.250 & 0.0007528 & 0.0004 & 3.278 & 1 \\
\hline 0.1000 & 3.241 & 0.0006325 & 0.0004 & 3.278 & 1 \\
\hline 0.1500 & 3.232 & 0.0007528 & 0.0004 & 3.278 & 1 \\
\hline 0.2000 & 3.223 & 0.0005164 & 0.0004 & 3.278 & 2 \\
\hline 0.2500 & 3.215 & 0.0005164 & 0.0004 & 3.277 & 2 \\
\hline 0.3000 & 3.206 & 0.0007528 & 0.0004 & 3.277 & 2 \\
\hline 0.3500 & 3.197 & 0.0004082 & 0.0004 & 3.277 & 3 \\
\hline 0.4000 & 3.188 & 0.0005164 & 0.0004 & 3.276 & 3 \\
\hline 0.4500 & 3.180 & 0.0005164 & 0.0004 & 3.276 & 3 \\
\hline 0.5000 & 3.171 & 0.0005164 & 0.0004 & 3.276 & 3 \\
\hline 0.5500 & 3.162 & 0.0008165 & 0.0004 & 3.275 & 4 \\
\hline 0.6000 & 3.153 & 0.0005164 & 0.0004 & 3.276 & 4 \\
\hline 0.6500 & 3.145 & 0.0006325 & 0.0004 & 3.275 & 4 \\
\hline 0.7000 & 3.135 & 0.0005164 & 0.0004 & 3.274 & 4 \\
\hline 0.7500 & 3.128 & 0.0005164 & 0.0004 & 3.274 & 5 \\
\hline 0.8000 & 3.119 & 0.0005164 & 0.0004 & 3.274 & 5 \\
\hline 0.8500 & 3.110 & 0.0007528 & 0.0004 & 3.273 & 5 \\
\hline 0.9000 & 3.103 & 0.0013290 & 0.0006 & 0 & 100 \\
\hline 0.9440 & 0 & No aplica & No aplica & 0 & No aplica \\
\hline
\end{tabular}

Cuadro 8. Convertidor a 5V con alimentación de 4.2V

\begin{tabular}{|c|c|c|c|c|c|}
\hline Corriente I $(\mathrm{A})$ & $\mathrm{V}_{\text {prom }}(\mathrm{V})$ & $\mathrm{S}_{\mathrm{x}}(\mathrm{V})$ & Incertidumbre $( \pm \mathrm{V})$ & $\mathrm{V}_{\text {sim }}(\mathrm{V})$ & Error $(\%)$ \\
\hline 0.0000 & 5.178 & 0.0004083 & 0.0003 & 5.930 & 15 \\
\hline 0.1000 & 5.175 & 0.0005164 & 0.0004 & 5.125 & 1 \\
\hline 0.1500 & 5.159 & 0.0005164 & 0.0004 & 5.119 & 1 \\
\hline 0.2000 & 5.143 & 0.0005164 & 0.0004 & 5.114 & 1 \\
\hline 0.2500 & 5.13 & 0.0005164 & 0.0004 & 5.114 & 0.3 \\
\hline 0.3000 & 5.119 & 0.0005164 & 0.0004 & 5.114 & 0.1 \\
\hline 0.3500 & 5.11 & 0.0007528 & 0.0004 & 5.114 & 0.1 \\
\hline 0.4000 & 5.101 & 0.0005164 & 0.0004 & 5.111 & 0.2 \\
\hline 0.4500 & 5.095 & 0.0006325 & 0.0004 & 5.070 & 0.5 \\
\hline 0.5000 & 5.089 & 0.0009832 & 0.0005 & 5.072 & 0.3 \\
\hline 0.5500 & 5.083 & 0.0005164 & 0.0004 & 5.072 & 0.2 \\
\hline 0.6000 & 5.078 & 0.0005164 & 0.0004 & 5.072 & 0.1 \\
\hline 0.6500 & 5.071 & 0.0005164 & 0.0004 & 5.072 & 0.02 \\
\hline 0.7000 & 5.066 & 0.0006325 & 0.0004 & 5.072 & 0.1 \\
\hline 0.7500 & 5.06 & 0.0005164 & 0.0004 & 5.072 & 0.2 \\
\hline 0.8000 & 5.052 & 0.0008165 & 0.0004 & 5.072 & 0.4 \\
\hline 0.8500 & 5.042 & 0.0005164 & 0.0004 & 5.072 & 0.6 \\
\hline 0.9000 & 5.038 & 0.0006325 & 0.0004 & 5.071 & 0.7 \\
\hline
\end{tabular}




\begin{tabular}{|c|c|c|c|c|c|}
\hline Corriente I $(\mathrm{A})$ & $\mathrm{V}_{\text {prom }}(\mathrm{V})$ & $\mathrm{S}_{\mathrm{x}}(\mathrm{V})$ & Incertidumbre $( \pm \mathrm{V})$ & $\mathrm{V}_{\text {sim }}(\mathrm{V})$ & Error $(\%)$ \\
\hline 0.95 & 5.029 & 0.0005164 & 0.0004 & 5.072 & 0.9 \\
\hline 1.00 & 5.024 & 0.0004082 & 0.0003 & 5.072 & 1 \\
\hline 1.05 & 5.02 & 0.0005164 & 0.0004 & 5.073 & 1 \\
\hline 1.10 & 5.012 & 0.0005164 & 0.0004 & 5.070 & 1 \\
\hline 1.15 & 5.004 & 0.0009832 & 0.0005 & 5.068 & 1 \\
\hline 1.20 & 4.999 & 0.0005164 & 0.0004 & 5.070 & 1 \\
\hline 1.25 & 4.988 & 0.0005164 & 0.0004 & 5.073 & 2 \\
\hline 1.30 & 4.985 & 0.0005164 & 0.0004 & 5.072 & 2 \\
\hline 1.35 & 4.981 & 0.0005164 & 0.0004 & 5.072 & 2 \\
\hline 1.40 & 4.51 & 0.0005164 & 0.0004 & 5.072 & 13 \\
\hline 1.45 & 4.22 & 0.0005164 & 0.0004 & 5.072 & 20 \\
\hline 1.50 & 3.709 & 0.0006325 & 0.0004 & 5.070 & 37 \\
\hline 1.55 & 3.684 & 0.0004082 & 0.0003 & 5.072 & 39 \\
\hline 1.60 & 3.662 & 0.0005164 & 0.0004 & 5.072 & 39 \\
\hline 1.65 & 3.636 & 0.0005164 & 0.0004 & 5.072 & 40 \\
\hline 1.70 & 3.61 & 0.0005164 & 0.0004 & 5.071 & 40 \\
\hline 1.75 & 3.58 & 0.0005164 & 0.0004 & 5.072 & 42 \\
\hline 1.80 & 3.545 & 0.0007528 & 0.0004 & 5.072 & 43 \\
\hline 1.85 & 3.504 & 0.0009832 & 0.0005 & 5.072 & 45 \\
\hline 1.90 & 3.441 & 0.0005164 & 0.0004 & 5.073 & 47 \\
\hline 1.95 & 3.321 & 0.0007528 & 0.0004 & 5.072 & 53 \\
\hline 1.962 & 0 & No aplica & No aplica & 5.072 & No aplica \\
\hline & & & & & \\
\hline
\end{tabular}

Cuadro 9. Convertidor a 5V con alimentación de 3V

\begin{tabular}{|c|c|c|c|c|c|}
\hline Corriente I $(\mathrm{A})$ & $\mathrm{V}_{\text {prom }}(\mathrm{V})$ & $\mathrm{S}_{\mathrm{x}}(\mathrm{V})$ & Incertidumbre $( \pm \mathrm{V})$ & $\mathrm{V}_{\text {sim }}(\mathrm{V})$ & Error $(\%)$ \\
\hline 0.0000 & 5.161 & 0.0007528 & 0.0004 & 5.930 & 15 \\
\hline 0.1000 & 5.145 & 0.0005164 & 0.0004 & 5.122 & 1 \\
\hline 0.1500 & 5.132 & 0.0005164 & 0.0004 & 5.123 & 1 \\
\hline 0.2000 & 5.122 & 0.0009832 & 0.0005 & 5.123 & 1 \\
\hline 0.2500 & 5.113 & 0.0005164 & 0.0004 & 5.118 & 0.3 \\
\hline 0.3000 & 5.106 & 0.0006325 & 0.0004 & 5.114 & 0.1 \\
\hline 0.3500 & 5.1 & 0.0005164 & 0.0004 & 5.111 & 0.1 \\
\hline 0.4000 & 5.094 & 0.0005164 & 0.0004 & 5.070 & 0.2 \\
\hline 0.4500 & 5.088 & 0.0007528 & 0.0004 & 5.072 & 0.5 \\
\hline 0.5000 & 5.082 & 0.0005164 & 0.0004 & 5.072 & 0.3 \\
\hline 0.5500 & 5.076 & 0.0007528 & 0.0004 & 5.072 & 0.2 \\
\hline 0.6000 & 5.07 & 0.0006325 & 0.0004 & 5.072 & 0.1 \\
\hline 0.6500 & 5.064 & 0.0004082 & 0.0003 & 5.072 & 0.02 \\
\hline 0.7000 & 5.053 & 0.0005164 & 0.0004 & 5.070 & 0.1 \\
\hline 0.7500 & 5.049 & 0.0007528 & 0.0004 & 5.072 & 0.2 \\
\hline 0.8000 & 5.043 & 0.0005164 & 0.0004 & 5.072 & 0.4 \\
\hline
\end{tabular}




\begin{tabular}{|c|c|c|c|c|c|}
\hline Corriente I $(\mathrm{A})$ & $\mathrm{V}_{\text {prom }}(\mathrm{V})$ & $\mathrm{S}_{\mathrm{X}}(\mathrm{V})$ & Incertidumbre $( \pm \mathrm{V})$ & $\mathrm{V}_{\text {sim }}(\mathrm{V})$ & Error $(\%)$ \\
\hline 0.8500 & 5.037 & 0.0006325 & 0.0004 & 5.072 & 0.6 \\
\hline 0.9000 & 5.032 & 0.0005164 & 0.0004 & 5.072 & 0.7 \\
\hline 0.9500 & 5.03 & 0.0007528 & 0.0004 & 5.071 & 0.9 \\
\hline 1.000 & 5.024 & 0.0005164 & 0.0004 & 5.073 & 1 \\
\hline 1.050 & 5.015 & 0.0007528 & 0.0004 & 5.067 & 1 \\
\hline 1.100 & 5.01 & 0.0006325 & 0.0004 & 5.066 & 1 \\
\hline 1.150 & 5.004 & 0.0007528 & 0.0004 & 5.073 & 1 \\
\hline 1.200 & 4.997 & 0.0007528 & 0.0004 & 5.071 & 1 \\
\hline 1.250 & 4.99 & 0.0007528 & 0.0004 & 5.073 & 2 \\
\hline 1.280 & 0 & No aplica & No aplica & 5.073 & No aplica \\
\hline
\end{tabular}

Finalmente en el cuadro 10, se muestran los resultados obtenidos por medio del segundo método de medición y se comparan con la simulación, la cual posee todos los componentes que se han podido incorporar a la simulación.

En esta comparación se identifican errores por debajo del 10\%, que permite considerar la simulación como una herramienta de diseño cuando no se requiere comprobar el valor exacto de la salida. Sin embargo, la simulación en PSpice brinda la posibilidad de obtener una buena respuesta para el diseño de las regulaciones y detectar errores en la elección de valores de los componentes necesarios para construir las configuraciones deseadas.

Cuadro 10. Comparación de los resultados utilizando el segundo método de medición.

\begin{tabular}{|c|c|c|c|c|}
\hline Vprom (V) & Sx (V) & Incertidumbre $( \pm \mathrm{V})$ & Vsim $(\mathrm{V})$ & Error $(\%)$ \\
\hline 3.278 & 0.0004 & 0.0003 & 3.364 & 3 \\
\hline 5.000 & 0.0004 & 0.0003 & 5.383 & 8 \\
\hline
\end{tabular}




\section{Conclusiones}

Se identificaron los componentes electrónicos que forman parte del sistema eléctrico de potencia utilizado en los nanosatélites del proyecto BIRDS 2 y se realizaron diagramas de bloques a distintos niveles que ubican los componentes en las etapas de funcionamiento para este sistema eléctrico de potencia.

Para el análisis del sistema eléctrico de potencia del proyecto BIRDS 2, se identifican y establecen como parámetros críticos las tensiones a la salida de los convertidores TPS61235, TPS63020 y la corriente que demanda la carga a estos convertidores, para niveles de carga de la batería de 3V y $4.2 \mathrm{~V}$.

Se logró simular el comportamiento de las tensiones a la salida de los convertidores TPS61235 y TPS63020, frente a los cambios de corriente exigidos por una carga electrónica con niveles de carga de la batería de 3V y 4.2V.

Se analizó la validez de la simulación del sistema eléctrico de potencia por medio de su comparación con valores obtenidos en las mediciones del modelo de ingeniería del sistema eléctrico de potencia de BIRDS 2, bajo las mismas condiciones de corriente en la carga y tensión de la batería. Se comparan estos datos por medio del cálculo de porcentajes de error, con lo que se determina que las tensiones a la salida de los convertidores TPS61235 y TPS63020 en la simulación son bastante exactas, excepto para el TPS61235 cuando la corriente de la carga no se encuentra entre $0.1000 \mathrm{~A}$ y $1.400 \mathrm{~A}$.

\section{Referencias}

[1] International Academy of Astronautics, Definition and Requirements of Small Satellites Seeking Low-Cost and Fast-Delivery, C. Mengu y G. Filippo, Edits., 2017.

[2] M. Patel, Spacecraft Power Systems, CRC Press, 2005.

[3] Laboratory of Spacecraft Environment Interaction Engineering, «Introduction,» 5 Septiembre 2018. [En línea]. Available: http://laseine.ele.kyutech.ac.jp/english/intro.html.

[4] G. Umberto, S. Luigi y D. L. Antonio, «Overcurrent Protection Performance Evaluation,» Space Power, Proceedings of the Sixth European Conference, $n^{\circ}$ 6, p. 411, 2002.

[5] N. Mohan, Power Electronics A First Course, Don Fowley, 2012.

[6] «Characteristics And Applications Of Semiconductor Power Devices : Power MOSFET And IGBT Transistors,» [En línea]. Available: https://steemit.com/steemstem/@biomanu/. [Último acceso: 20 Agosto 2018].

[7] H. Rashid, Electrónica de Potencia: Circuitos, dispositivos y aplicaciones, Prentince Hall, 1993.

[8] A. Devices, «LTSpice,» [En línea]. Available: http://www.analog.com/en/design-center/design-tools-and-calculators/ltspice-simulator.html?domain=www.linear.com. [Último acceso: 30 Agosto 2018].

[9] L. Electronics, «Proteus: Commercial Price List,» [En línea]. Available: https://www.labcenter.com/pricing/ comm/. [Último acceso: 30 Agosto 2018].

[10] C. Quintáns, Simulación de circuitos electrónicos con OrCAD 16 Demo, Digitalia, 2008.

[11] K. Olav, «Design and Implementation of the Electrical Power System for the CubeSTAR Satellite,» University of Oslo, 2013.

[12] D. Venegas, N. Gutiérrez y D. Murillo, Manual de Laboratorio: Laboratorio de Física General I, Tecnológico de Costa Rica, 2013.

[13] H. Fink y G. Wayne, Power Electronics Circuits, Devices, and Applications, McGraw Hill, 2012. 